\title{
Especies de mosca de la fruta Diptera: Tephritidae, presentes en plantas frutícolas hospederas de la Provincia de Rodríguez de Mendoza, Amazonas 2016
}

\section{Species of fruit fly Diptera: Tephritidae, present in host fruit plants of the Province of Rodríguez de Mendoza, Amazonas region 2016}

\author{
Ancelmo López, Santos Leiva-Espinoza ${ }^{2}$
}

\section{RESUMEN}

Las plantaciones frutícolas ostentan diferentes problemas fitosanitarios, dentro de los cuales la mosca de la fruta es la plaga principal y puede causar la destrucción completa de los frutos donde se hospeda. El presente trabajo de investigación tuvo como objetivo identificar las especies de mosca de la fruta DÍPTERA: TEPHRITIDAE, presentes en las principales plantas frutícolas hospederas de la provincia de Rodríguez de Mendoza - Perú. Se determinó el número de plantas frutícolas hospederas de los 11 distritos de esta provincia, recolectándose además, muestras de frutos maduros con aparente infestación con huevos y/o larvas para así recuperar adultos de mosca de la fruta a través de crianzas in-situ y ex-situ, realizando posteriormente la identificación de los individuos recuperados y a través del uso de llaves y comparaciones morfológicas propuestas por Tigrero 1998. Los principales hospederos de mosca de la fruta que son: Psidium guajava, Citrus sinensis, Inga edulis, Quararibea cordata y Pouteria caimito, Hospederos en los que estuvieron presentes las siguientes especies de mosca de la fruta: Anastrepha fraterculus (en naranja, guayaba y guaba), Anastrepha distincta (en naranja y guaba), Anastrepha serpentina (en caimito y zapote), Anastrepha striata y Anastrepha ornata (en guayaba).

Palabras clave: hospedero, mosca de la fruta.

\begin{abstract}
Fruit plantations boast different phytosanitary problems, within which the fruit fly is the main pest and can cause the complete destruction of the fruits where it is housed. The objective of this research work was to identify the fruit fly species Diptera: Tephritidae, present in the main host fruit plants of the province of Rodríguez de Mendoza - Peru. The number of host fruit plants of the 11 districts of this province was determined, also collecting samples of ripe fruits with apparent infestation with eggs and / or larvae to recover fruit fly adults through in-situ and ex-in- situ breeding. -situ, subsequently carrying out the identification of recovered individuals and through the use of keys and morphological comparisons proposed by Tigrero 1998. The main fruit fly hosts that are: Psidium guajava, Citrus sinensis, Inga edulis, Quararibea cordata and Pouteria caimito, Hosts in which the following species of fruit fly were present: Anastrepha fraterculus (in orange, guava and guava), Anastrepha distincta (in orange and guaba), Anastrepha serpentina (in caimito and zapote), Anastrepha striata and Anastrepha ornata (in guayaba)
\end{abstract}

Keywords: host, fruit fly.

\footnotetext{
${ }^{1}$ Bachiller en Ingeniería Agrónoma. Universidad Nacional Toribio Rodríguez de Mendoza de Amazonas. Correo electrónico: ancelopo_91@hotmail.com

${ }^{2}$ Ingeniero Agrónomo. Universidad Nacional Toribio Rodríguez de Mendoza de Amazonas. Correo electrónico: santos.leiva@untrm.edu.pe
} 


\section{INTRODUCCIÓN}

En las plantaciones frutales se encuentran problemas fitosanitarios que vienen mermando la producción, siendo la mosca de la fruta el principal problema de carácter entomológico, éstas se hallan distribuidas en áreas tropicales y subtropicales de todo el mundo (González, 2013). Son insectos del orden Diptera de la familia Tephritidae, consideradas plagas clave y cuyo daño principal es ocasionado por la larva en su proceso de alimentación, generando pudrición o mala apariencia de la fruta, convirtiéndose en caldo de cultivo para los microorganismos patógenos que aceleran la descomposición de las frutas, estas ocasionan: disminución de la producción, aumento de los costos de producción, disminución del valor comercial y restricciones en el acceso a los mercados externos. (ICA, 2012).

Las organizaciones como: la FAO, la OMS y la OCDE han visto la necesidad de establecer de manera urgente las estrategias para la protección fitosanitaria en cada país a fin de reducir al mínimo el riesgo fitosanitario, que apoyará a los productores (Iñiguez Luzuriaga, 2015).

Las frutas hospederas de la mosca de la fruta son de distintas especies. Tigrero (2009) encontró en Psidium guajava, Annona cherimola, Pouteria lúcuma a Anastrepha fraterculus. Gordillo y Pizarro (2016) reconocieron a las siguientes frutas como hospederos: Naranja, Chirimoya, guayaba. Guaba, nogal, mandarina, durazno; descubriendo ocho especies de Anastrapha: A. fraterculus Wied., A. distincta Greene, A. grandis Macquart, A. striata Schiner, A. obliqua Macquart, A. serpentina Wied., A. spp. y Ceratitis capitata Wied. Las especies vegetales más atacadas por especímenes de moscas de la fruta son: guayaba, naranja, durazno, guaba. (Vilatuña, 2016).

La mosca de la fruta es un organismo muy dinámico, con fácil poder de adaptación, encontrado en los predios frutícolas, condiciones óptimas para su desarrollo y multiplicación. El Perú cuenta con una producción frutícola dispersa, no organizada, no planificada, que ha provocado el incremento y la presencia de varias especies de moscas de la fruta (Agrocalidad, 2013).

En la provincia Rodríguez de Mendoza, existen condiciones de clima y suelo muy favorables para el cultivo de frutales y aunque su producción se limita a pequeñas plantaciones de huerto, éstos simbolizan, en mucho de los casos pequeñas fuentes de ingresos económicos para muchas familias de la provincia; entre estas especies existentes, podemos citar: la chirimoya, los cítricos, piña, guaba, guayaba, zapote y otros frutales; sin embargo, en la actualidad, la fruta de estas plantaciones, vienen siendo afectados por altos niveles de incidencia de mosca de la fruta, los mismos que causan pudrición y destrucción de los frutos próximos a la cosecha.

La presente investigación tuvo como objetivo identificar las especies de mosca de la fruta Diptera: Tephritidae, presentes en plantas frutícolas hospederas de la Provincia de Rodríguez de Mendoza, Región Amazonas 2016.

\section{MATERIALES Y MÉTODOS}

La investigación se realizó en once distritos de esta provincia Rodríguez de Mendoza: Limabamba, Totora, Chirimoto, Milpuc, Santa Rosa, San Nicolás, Mariscal Benavidez, Omia, Longar, Cochamal y Huambo; dicha provincia se encuentra a una altitud promedio de $1595 \mathrm{msnm}$., la temperatura media anual $21^{\circ} \mathrm{C}$, y la precipitación de 1467,8 mm/año.

La población estuvo representada por la sumatoria de plantas frutícolas de cada especie frutal de los once sectores de estudio. Para determinar las especies de frutales existentes en la zona, se realizó encuestas en los hogares de cada punto de estudio para determinar ¿Cuáles? y ¿cuántos? son los principales frutales posibles hospederos de mosca de la fruta existentes en cada sector de los distritos de la Provincia Rodríguez de Mendoza.

La identificación de las especies de moscas de la fruta, se inició con la recolección de fruta según estación. Para lo cual se realizó el muestreo correspondiente, recolectando al azar y tomando de la planta, un fruto por cada árbol hasta completar 02 $\mathrm{Kg}$. De fruto con indicios de haber sido atacados por mosca de la fruta. Dichas muestras fueron colocadas en bolsas de polietileno previamente rotuladas y preparadas para la crianza in-situ y ex-situ.

Se colectaron un total de $88 \mathrm{~kg}$ de fruta (naranja, guayaba, guaba, caimito y zapote).

La crianza in-situ se realizó en cada lugar donde se recolectó la fruta; las muestras que se colectaron fueron colocadas en canastas plásticas de $60 \mathrm{~cm}$ de largo por $35 \mathrm{~cm}$ de ancho y $33 \mathrm{~cm}$ de alto, las que se acondicionaron previamente con tierra suelta esterilizada (superficie de empupado) para posteriormente y luego de que las frutas fueran lavadas con hipoclorito de sodio al $2 \%$ y colocadas sobre la superficie de empupado, éstas se cubrieron con una malla antiáfida para proteger de agentes externos y evitar que los adultos eclosionados puedan 
escapar.

La crianza ex-situ se realizó en el Laboratorio de Sanidad Vegetal de la Universidad Nacional Toribio Rodríguez de Mendoza, aquí también se utilizaron canastas plásticas y se procedió con la crianza de la misma manera que lo efectuado en la crianza in-situ.

Una vez obtenidos los adultos de mosca de la fruta, tanto de la crianza in-situ como ex-situ, los individuos fueron colocados en unos frascos de 20 $\mathrm{cm}^{3}$ con alcohol a $70^{\circ}$ para conservar las estructuras y coloración de cada especímen; las muestras fueron acondicionadas especialemnte para ser observados en un esteremicroscopio y así realizar la captura de imágenes fotográficas de cada estructura del insecto, clave para su identificación.

Para la Identificación morfológica de especies de mosca de la fruta; las características y llaves encontradas, fueron comparadas con el manual de "Tigrero - 1998".

Una vez Identificadas las especies de mosca de la fruta, se implementó una colección de referencia, dispuesta en una caja entomológica de madera y vidrio, donde se disponen los especímenes recuperados. Dicha caja entomológica fue depositada en el Laboratorio de Sanidad Vegetal de la Universidad Nacional Toribio Rodríguez de Mendoza.

\section{RESULTADOS}

\subsection{Población de frutales hospederos de mosca de la fruta.}

En el ámbito de estudio del presente trabajo, se encontraron plantas de espacies frutales en forma aislada y de crecimiento natural, así como también algunos pequeños huertos comerciales instalados por productores aficionados a la fruticultura.

Las encuestas reportan la presencia de las siguientes especies frutales: Guayaba (Myrtaceae), naranja (Rutáceas), guaba (Fabaceae), zapote (Malvaceae) y caimito (Sapotaceae); siendo este último el de menor población en todos los distritos inmersos en el presente estudio.

Los frutales de naranja, caimito y zapote están presentes como un árbol frutal aislado de función secundaria dentro de agroecosistemas diversos, mientras que las plantas de guaba cumplen el rol de sombra para el cultivo de café. La guayaba es un frutal nativo en toda la provincia Rodríguez de Mendoza, encontrándose plantas en los bordes de los caminos, en los pastizales y dentro de los bosques.
De todas las especies frutales, el $100 \%$ presenta elevados niveles de ataque de mosca de la fruta, siendo la guayaba el frutal más afectado por esta plaga.

Tabla 1. Especies frutales y población presente en los sectores de estudio.

\begin{tabular}{|c|c|c|c|c|c|}
\hline & \multicolumn{5}{|c|}{$\begin{array}{l}\text { Número de plantas frutales por sector } \\
\text { de estudio }\end{array}$} \\
\hline & Naranja & Guayab & Caimito & Zapote & Guaba \\
\hline Cochamal & 150 & 120 & 21 & $\mathrm{NE}$ & 73 \\
\hline Longar & 65 & 36 & $\mathrm{NE}$ & $\mathrm{NE}$ & 62 \\
\hline $\begin{array}{l}\text { Mariscal } \\
\text { Benavides }\end{array}$ & 74 & 135 & 13 & 4 & 58 \\
\hline San Nicolás & 58 & 102 & 4 & 2 & 74 \\
\hline Huambo & 67 & 202 & 14 & $\mathrm{NE}$ & 85 \\
\hline Santa Rosa & 14 & 16 & 1 & $\mathrm{NE}$ & 54 \\
\hline Totora & 32 & 134 & NE & $\mathrm{NE}$ & 98 \\
\hline Limabamba & 38 & 112 & 16 & $\mathrm{NE}$ & 88 \\
\hline Chirimoto & 47 & 132 & 14 & $\mathrm{NE}$ & 78 \\
\hline Milpuc & 22 & 98 & $\mathrm{NE}$ & $\mathrm{NE}$ & 56 \\
\hline Omia & 18 & 32 & 3 & $\mathrm{NE}$ & 76 \\
\hline
\end{tabular}

NE: No existente.

\subsection{Identificación de especies de mosca de la fruta}

Especie recuperada de hospederos de Naranja Citrus sinensis y Guaba Inga edulis
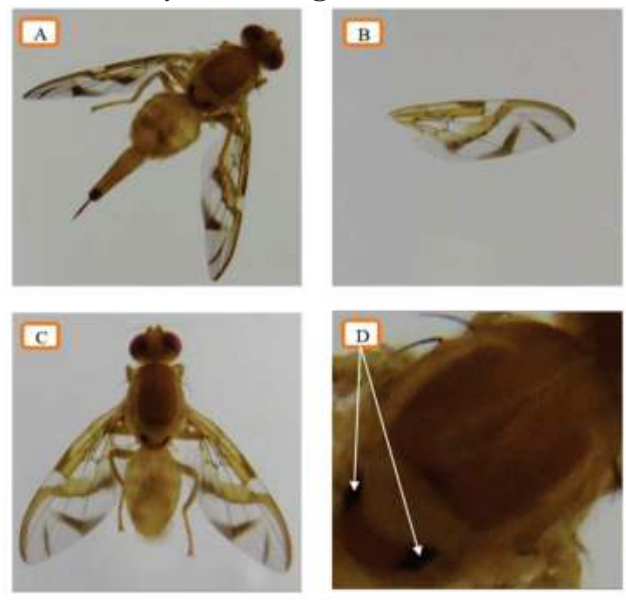

Figura2. (A) Adulto hembra, (B) Ala anterior mostrando máculas típicas del genero Anastrepha, (C) Adulto macho recién eclosionado, (D) Características del tórax. 
La especie observada es de tamaño medio, y entre otras características se observa un color amarillo claro, tórax y abdomen de color amarillo uniforme, funda del ovipositor más larga que el abdomen. Además, en las imágenes se observa:

\section{(A) Imagen de mosca de la fruta hembra.}

(B) Vista general del ala: banda Costal y S ligeramente unidas en la vena $\mathrm{R} 4+5$, bandas $\mathrm{V}$ completa, poco separada con la banda en $\mathrm{S}$

(C) Imagen de mosca de la fruta macho

(D) Tórax, Scutum con un pequeño punto infuscado en la sutura scuto-scutelar; pero también este puede ser claramente definido o ausente. El Sub-escutelo con manchas negruzcas a cada lado.

Especie recuperada de hospederos de Citrus sinensis, Inga edulis y Psidium guajava
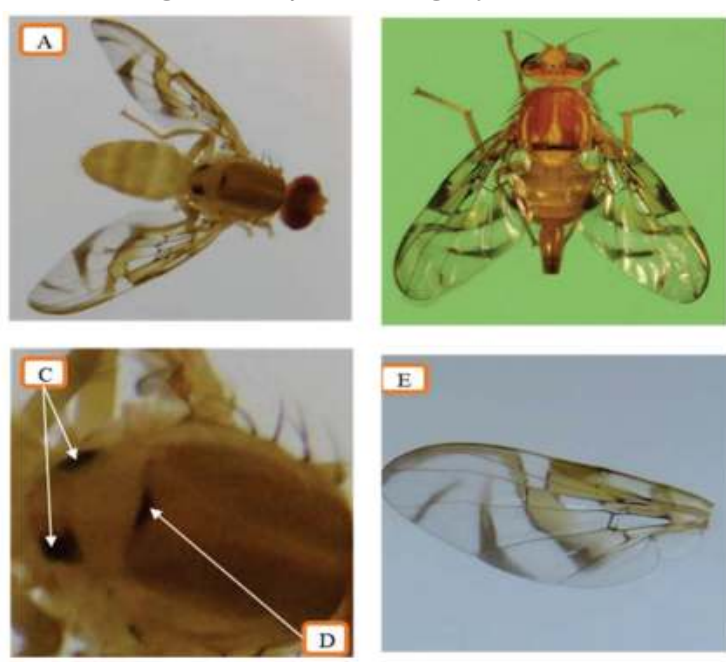

Figura 3. (A) Adulto macho, (B) Adulto hembra con ovipositor tubular, (C, D) Características del tórax, (E) Característica del ala anterior.

La figura 3 muestra las siguientes características: Especie de tamaño medio de color café amarillento, funda del ovipositor más corta que el abdomen, además de otras características como:

(A) Imagen del macho de mosca de la fruta.

(B) Hembra adulta de mosca de la fruta

(C) y (D) Tórax: Banda longitudinal de color claro que se ensancha hacia la parte posterior; punto de color negro localizado en la parte central de la sutura escuto-escutelar, generalmente está bien definido. Sub-escutelo con una mancha a cada lado que se extiende al medioterguito.

(E) Vista general del ala: bandas costal y "S" ampliamente unidas en la vena $\mathrm{R} 4+5$ y la vena en $\mathrm{V}$ generalmente separada de la banda en $\mathrm{S}$.

Especies recuperadas en hospedero de guayaba (Psidium guajava).
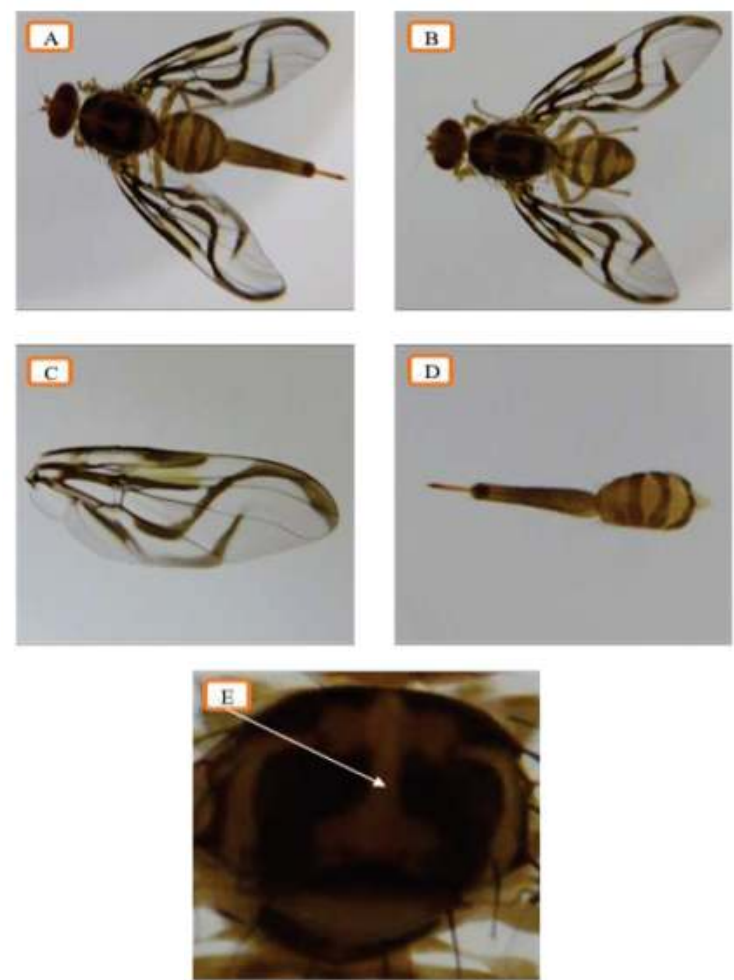

Figura 4. (A) Adulto hembra, (B) Adulto macho, (C) Ala posterior, (D) Ovipositor y abdomen, (E) Característica del tórax.

Los parámetros morfológicos observados en los individuos recuperados de las crianzas, encontrados en el hospedero Guayaba, son los siguientes: Especie de tamaño medio a grande, tórax y abdomen de coloración mayormente oscuro, funda del ovipositor más largo que el abdomen. La figura 4 muestra los siguientes detalles:

(A) Hembra adulta. Funda del ovipositor más larga que el abdomen

(B) Macho adulto

(C) Vista general del ala: bandas costal y "S" desconectados entre sí; banda en $\mathrm{S}$ completa; banda en $\mathrm{V}$ con el brazo externo ausente, brazo interno largamente proyectado hacia atrás hasta unirse o casi unirse a la parte posterior de la banda en S.

(D) Ovipositor más largo que el abdomen, manchas claras y anchas transversales en el abdomen.

(E) Tórax, en la parte dorsal central del tórax se observa una mancha clara longitudinal y dos manchas laterales que se proyectan desde el mesotórax hasta la sutura scuto-scutelar. 
Especies recuperadas en hospederos de Pouteria caimito y Quararibea cordata
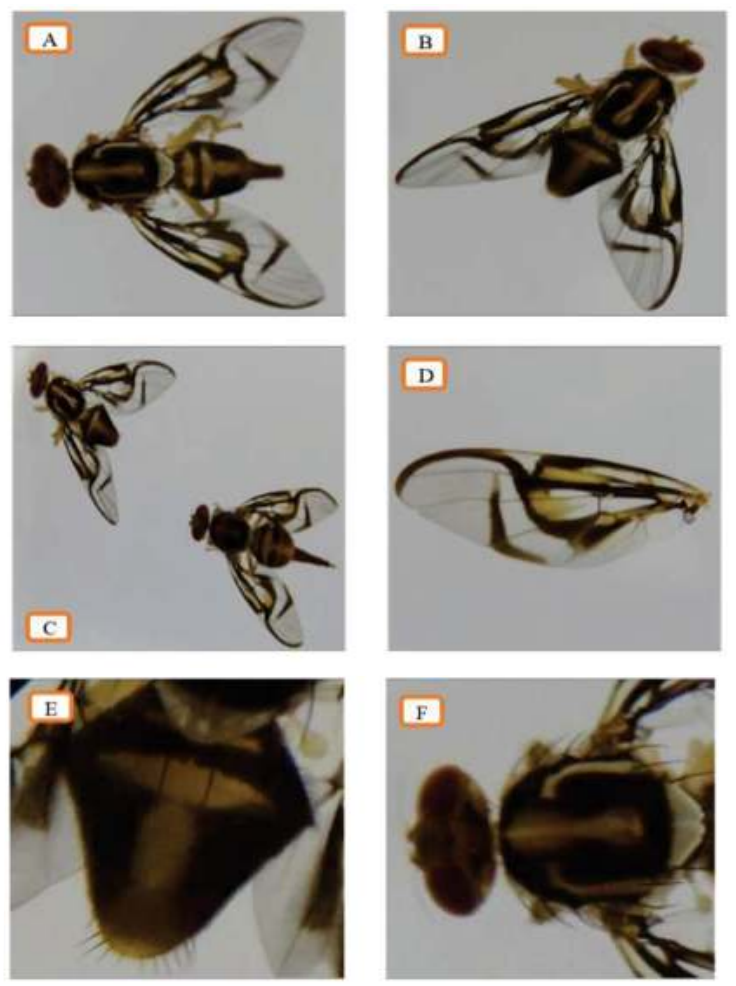

Figura 5. (A) Adulto hembra, (B) Adulto macho, (C) Adultos de Macho y Hebra, (D) Bandas alares, (E) Vista dorsal del abdomen, (F) Tórax.

Según se observa en la figura 5, las imágenes permiten observar que la especie posee las siguientes características:

Especie de tamaño medio, cuerpo de coloración mayormente marrón oscuro, la característica principal es que presenta una mancha clara en forma de $\mathrm{T}$ e lo largo dorsal del abdomen.

(A) Mosca adulta hembra.

(B) Mosca adulta macho.

(C) Macho y Hembra adultos.

(D) Vista general del ala: bandas alares de color castaño negruzco, bandas Costal y $\mathrm{S}$ delgadas conectadas entre sí, en la vena $\mathrm{R} 4+5$; la banda en V incompleta, solo presenta el brazo interno que es delgado y separado de la banda en $\mathrm{S}$.

(E) Vista dorsal del abdomen, se observa una mancha clara en forma de $\mathrm{T}$ a lo largo de los segmentos abdominales (esta es una característica clave muy importante para la identificación de Anastrepha serpentina)
(F) Tórax: Dorso del tórax café oscuro con manchas amarillas; en el mesonoto se observan bandas de color negro en forma de "U" con una interrupción a la altura de la sutura transversal y con otra banda más angosta a cada lado de los brazos en "U", de color oscuro y en posición lateral al mesonoto.

Especies recuperadas en hospederos de Psidium guajava
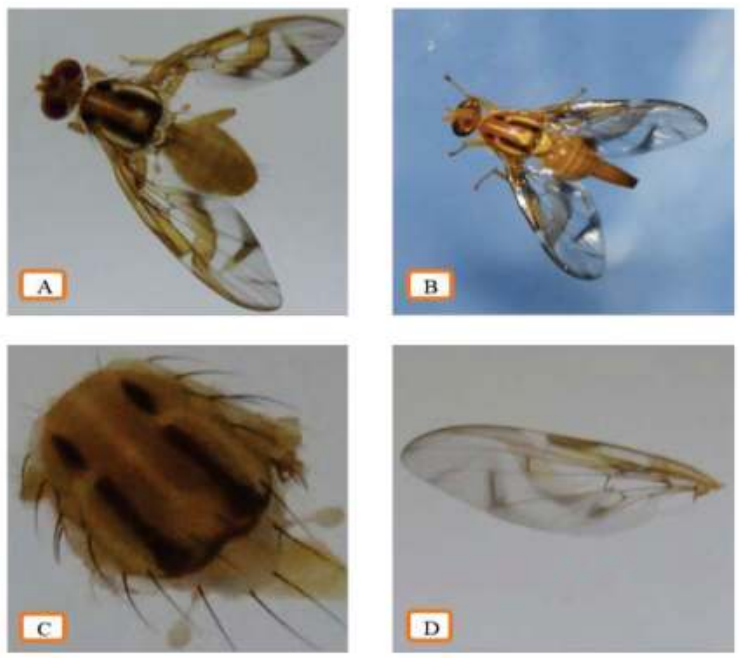

Figura 6. (A) Adulto macho, (B) Adulto hembra, (C) Características del tórax, (D) Bandas alares.

La figura 6 muestra las imágenes que corresponden a los adultos de mosca de la fruta recuperados de la "guayaba"(Psidium guajava) presenta las características siguientes:

Especie de tamaño medio con un patrón de coloración marrón amarillento, funda del ovipositor más larga que el abdomen y muy corpulenta en su base; detallándose cada estructura de identificación:

(A) Macho de mosca de la fruta adulto

(B) Hembra de mosca de la fruta adulta

(C) Tórax: scutum con bandas laterales negras, anchas y cubiertas de micro setas, las bandas longitudinales amplias de color negro, formando una "U".

(D) Vista general del ala: bandas Costal y $\mathrm{S}$ siempre unidas de manera amplia, bandas $\mathrm{SyV}$ siempre separadas. 


\section{DISCUSIONES}

La provincia Rodríguez de Mendoza presenta un clima templado, que se encuentra a una altitud promedio de $1595 \mathrm{msnm}$, la temperatura media anual $21 \mathrm{C}^{\circ}$, la precipitación es $1467,8 \mathrm{~mm} /$ año (Instituto de Investigación para el Desarrollo Sustentable de Ceja de Selva, 2017); siendo estos factores climatológicos determinantes para la existencia de plantas frutícolas; por lo que se encontró que en todos los distritos, están presentes los frutales como: Psidium guajava (Myrtaceae), Citrus sinensis (Rutaceae), Inga edulis (Fabaceae), Pouteria caimito (Sapotaceae) y Quararibea cordata (Malvaceae). Del mismo modo Cuculiza y Torres, 1975 en Huánuco - Perú, reportaron la presencia de guayaba, cítricos (naranja), chirimoya, pacae y café a pesar que Huánuco se encuentra en altitudes entre 1800 a $2200 \mathrm{msnm}$, con temperatura que oscila entre 19 y 20 $\mathrm{C}^{\circ}$; ante ello se puede discutir que los frutales antes mencionados presentan una amplia adaptabilidad, encontrándose en diferentes rangos altitudinales y al mismo tiempo son hospederos de la mosca de la fruta.

En el presente estudio, se encontró que los principales hospederos de mosca de la fruta fueron: guayaba, naranja, guaba, caimito y zapote; al respecto Cuculiza y Torres (1975) en Valle de Huánuco mencionan que los principales frutales hospederos de moscas de la fruta es el guayabo, siguiendo en orden de importancia el café (exclusivo de Ceratitis), luego el naranjo, chirimoyo y por último el pacae (exclusivo para Anastrepha distincta) y algo de Ceratitis; por otro lado en Ecuador Tigrero (2009) reportó que los hospederos más importantes son la guayaba, de 7 especies de moscas de la fruta; en Chirimoya 6 especies y en lúcuma de 6 especies. De ese modo lo encontrado en el presente trabajo tendría coincidencia con los trabajos realizados en otros lugares, en función a los hospederos más afectados por la mosca de la fruta.

Con respecto a la identificación de especies de mosca de la fruta, Los resultados encontrado así como el uso de claves de naturaleza morfológica utilizados en el presente trabajo, han permitido determinar que para el caso de las especies recuperadas de hospederos de Naranja, las especies recuperadas corresponden a las especies Anastrepha distincta y Anastrepha fraterculus; del hospedero Guaba (Inga edulis), la especie Anastrepha distincta, Anastrepha fraterculus. Por otro lado, en el hospedero "guayaba" (Psidium guajava), se determina que la especie corresponde a Anastrepha fraterculus, Anastrepha ornata y Anastrepha striata. Finalmente, las características morfológicas de los adultos recuperados del "caimito" (Pouteria caimito) y del "zapote"(Quararibea cordata), corresponde a la especie de Tephritidae Anastrepha serpentina. Los resultados son corroborados cuando Vilatuña, et al., (2016), sostiene que estas especies de fruta son las más atacadas por las especies referenciadas renglones atrás.

La crianza In-situ de mosca de la fruta permite evaluar en sus condiciones ambientales originales: el ciclo biológico de la especie, los días transcurridos de cada estadío, así como también el número de especímenes adultos recuperados de una determinada cantidad de frutos. En el presente trabajo, la crianza in-situ determinaron que, el mayor número de moscas adultas recuperadas a partir de $02 \mathrm{Kg}$ de fruta fue de Psidium guajava, de lo cual podríamos decir que este es el hospedero más apetecible para la mosca de la fruta encontrada en la Provincia Rodríguez de Mendoza. Por otro lado, el menor número de especímenes de mosca de la fruta recuperados de la crianza in-situ fue de Pouteria caimito. Al respecto, Aluja, (1984) en México reporta que la guayaba es el frutal más afectado por la mosca de la fruta, especialmente por Anastrepha striata.

La especie Anastrepha fraterculus (Tephritidae) con mayor frecuencia se encontró en frutas Citrus sinensis, seguido en frutas de Inga edulis y finalmente se reporta a esta especie en una sola crianza en Psidium guajava, por lo que podría deducirse que Anastrepha fraterculus tendría preferencia por frutos de naranja, guaba y guayaba; de igual modo Gonzáles, et al., (2011) realizaron estudios en la Paz, Bolivia, reportando a Anastrepha fraterculus en frutos de naranja y guayaba; del mismo modo, Renata, Montes y Raga, (2014) en Brasil realizaron la colecta de los frutos de guayaba en el periodo de septiembre-abril, en la cual identificaron a Anastrepha fraterculus. Por lo que se concluye que la presente investigación concuerda con otras investigaciones en cuanto a la preferencia alimenticia de Anastrepha fraterculus.

En los sectores intervenidos en el presente estudio, la especie Anastrepha distincta (Tephritidae), fue recuperado de frutos de Inga edulis (guaba) y en menor cantidad en frutas de Citrus sinensis (naranja); por otra parte, Vilatuña, et al., (2016) en Ecuador menciona que las especies vegetales más atacadas por Anastrepha distincta fueron: guayaba, naranja, durazno y guaba; Por lo que nos da entender que Anastrepha distincta mantiene su preferencia alimenticia (guaba y naranja) tanto en Ecuador y en la provincia donde se desarrolló el estudio.

La temporada de fructificación y cosecha de guaba en 
la provincia Rodríguez de Mendoza son en los meses de enero a abril de cada año, por lo que, a fin de aprovechar el pico más alto de producción, las muestras para realizar crianza In-situ fueron colectados en el mes de febrero del año 2017; en dichas crianzas se recuperaron e identificaron dos especies de mosca de la fruta: Anastrepha fraterculus, Anastrepha distincta, ambas de la familia Tephritidae. Del mismo modo, Gordillo y Pizarro (2016) en Cuenca en el Ecuador encontraron a estas dos especies cuando colectaron sus muestras de frutas en el mes de Setiembre a diciembre. En este sentido podríamos discutir que Anastrepha fraterculus y Anastrepha distincta tienen preferencia por guaba en cualquier época del año.

De la crianza In-situ se recuperaron adultos de Anastrepha striata, solamente de la especie frutal de guayaba, lo cual podríamos indicar su especificidad y preferencia por esta fruta. Esto coincide con investigaciones realizadas por Renata, Montes y Raga (2014) en Brasil, que identificaron solamente a esta especie en su estudio en muestras de guayaba.

Por otro lado, en la presente investigación, se reporta la existencia de Anastrepha ornata (Tephritidae) únicamente presente en frutos de Psidium guajava y proveniente del distrito de Santa Rosa Provincia Rodríguez de Mendoza; al respecto, la investigación "Hospederos de la mosca de la fruta en Ecuador" realizado por Tigrero (2009) reportó a Psidium guajava como hospedero de Anastrepha ornata.

En diferentes investigaciones realizadas en Perú y otros países de Norte América se reporta que Anastrepha serpentina (Tephritidae) prefiere hospederos de Pouteria caimito (caimito) y Quararibea cordata (zapote); cabe mencionar que en la investigación realizada por Ariza-Flores (2009) en el estado de Morelos-México quienes realizaron la colección de muestras de frutas de caimito y zapote de altitudes promedio de $1600 \mathrm{msnm}$, del mismo modo Ragal (2003) en el Estado de San Paulo Brasil realizó identificación de mosca de la fruta en frutas de zapote y caimito, reportando a Anastrepha serpentina como especie especifica. De igual manera en el presente trabajo en la provincia Rodríguez de Mendoza, Amazonas - Perú, a pesar de la reducida población de plantas de caimito y zapote, se reporta a Anastrepha serpentina en todas las muestras colectadas de estos frutales.

Se recuperó e identificó la existencia de : 1) Anastrepha fraterculus, 2) Anastrepha distincta, 3) Anastrepha striata, 4) Anastrepha ornata y 5) Anastrepha serpentina en 05 hospederos diferentes: Citrus sinensis (naranja), Psidium guajava (guayaba), Inga edulis (guaba), Pouteria caimito y
Quararibea cordata (zapote); por otro lado Gordillo y Pizarro (2016) en la provincia de Azuay - Ecuador identificaron ocho especies de mosca de la fruta: Anastrapha fraterculus, Anastrapha distincta, Anastrapha grandis, Anastrapha. Striata, Anastrapha obliqua, Anastrapha serpentina, Anastrapha spp y Ceratitis capitata Wied. en frutales de Naranja, Chirimoya, guayaba. Guaba, nogal, mandarina, durazno. De lo mencionado anteriormente se discute que las especies Anastrepha fraterculus, Anastrepha distincta, Anastrepha striata y Anastrepha serpentina están presentes diferentes lugares del país y Norte América.

\section{CONCLUSIONES}

Todos los frutales evaluados en el presente trabajo de investigación (guayaba, naranja, guaba, caimito y zapote) son hospederos de la mosca de la fruta, estos frutales de estación coinciden en su periodo de maduración y cosecha durante los meses de enero a junio de cada año, época en que existen elevados niveles de incidencia de daños causados por la mosca de la fruta. La guayaba (Psidium guajava) resulto ser el frutal más afectado y mejor hospedero.

Se encontraron 05 especies de mosca de la fruta $=$ (TEPHRITIDAE DIPTERA) en 11 distritos de la Provincia Rodríguez de Mendoza, dichas especies de mosca de la fruta son las siguientes: Anastrepha distincta, Anastrepha fraterculus, Anastrepha ornata, Anastrepha serpentina y Anastrepha striata,

Se implementó una colección de referencia, cuyo depósito fue efectuado al museo de Entomología del Laboratorio de Sanidad Vegetal de la Universidad Nacional Toribio Rodríguez de Mendoza de Amazonas.

\section{REFERENCIAS BIBLIOGRAFICAS.}

Agrocalidad.(2013). Identificación de Anastrepha ludenns.Obtenidodecontent/uploads/downlo ads/2013/08/Anastrepha\%20ludens.pdf

Aluja, M. (1993). Manejo integrado de la mosca de la fruta(Vol. 1). México: Trilla.

Ángeles, J. (2003). Problemática en la comercialización de naranja (citrus sinencis) enelestadodeVeracrus.¿Obtenidodehttp://rep ositorio.uaaan.mx:8080/xmlui/bitstream/han dle/123456789/169/T13806\%20ANGELES $\% 20$ DEL $\% 20$ ROSAL $\% 2 C \% 20 J E S U S \% 20$ $\% 20$ TESIS.pdf?seq 
Cuculiza, M., y Torres, E. (1975). Mosca de la fruta en las principales plantas hospederas del valle Huánuco. Peruana de Entomología, 18(1).Obtenidodehttp://sisbib.unmsm.edu.p e/BVRevistas/entomologia/v18/pdf/a15v18. pdf

Gonzáles, M., Loza-Murguía, M., Hugh, S., Cuba, N., Almanza, J. C., y Ruiz, M. (2011). Dinámica poblacional de adultos de la mosca boliviana de la fruta Anastrepha sp. (Díptera: Tephritidae) en el Municipio de Coroico, Departamento de La Paz,

González, P. (2000). Problemas Entomológicos en los Frutales de Arequipa, Moquegua, Tacna. per de ent., 9(1), 16-23. Obtenido de http://sisbib.unmsm.edu.pe/BVRevistas/ent omologia/v09/pdf/a03v09.pdf

Gordillo, N., y Pizarro, F. (2016). Monitoreo de las especies y hospederos alternativos de los géneros Anastrepha y ceratitis en los cantones Gualaceo Chordeleg de Sigsig de la provincia de Azuay. Tesis. Obtenido de http://dspace.ucuenca.edu.ec/bitstream/123 456789/24022/1/TESIS\%20.pdf

ICA. (2010). Importancia de la mosca de la fruta en el mercado internacional de productos frescos.Obtenidodehttp://www.ica.gov.co/A reas/Agricola/Servicios/EpidemiologiaAgrícola/Documentos/Importancia_Moscas .aspx

Iñiguez, G. J. (2015). Caracterización e identificación de las especias de la mosca de la fruta presentes en los cultivos hortofrutícolas el cantón Chaguarramba. tesis.

Jaramillo, M., Benavides, P., Rendón, J. R., Montoya, D., Valencia, F., \& Trejos, J. (2015). Mosca de las frutas de la zona central cafetalera de Colombia y la zona ilustrada. C e n i c a fé, 66 ( 2 ), $58-72$. Obtenidodehttp://www.cenicafe. org/es/publications/4.LasMoscas.pdf

López, R. (2009). Cultivo frutículas con el potencial d e exportación. Obtenido de http://www.minagri.gob.pe/portal/download $/ \mathrm{pdf} /$ direccionesyoficinas/oficina_ap oyo enlace/cultivos fruticolas valle chillo n.pdf

RagaI, A., MachadoII, R., Costa, A., Souza, M., Ferraz, R., \& Saes, L. (2003). First occurrence of Anastrepha serpentina and Anastrepha leptozona (Dip.: Tephritidae) on abiu (Pouteria caimito) in the State of São Paulo, Brazil. Bras. Frutic., 25(3). doi:10.1590/S0100-29452003000200040

Renata, S., Montes, S., \& Raga, A. (2014). Fruit flies in a guava orchard in Indiana county, São Paulo,Brasil. IDESIA, 32(3). Obtenido de http://www.scielo.cl/pdf/idesia/v32n3/art13. pdf

Rodríguez, R., \& Uchôa, M. (2011). Species of fruit flies (Diptera: Tephritidae) in a transect of the Amazonian Rainforest in Oiapoque, Amapá, Brazil. Zoología, 28(5), 653-657. doi:10.1590/S1984-46702011000500013

Salmerón, A. L. (2016). Hymenoptera Parasitoides asociados a Anastrepha spp (Díptera: Tephritidae) en frutales de San Francisco Libre y El Jicaral, Nicaragua. Tesis. Obtenido d

http://repositorio.una.edu.ni/3449/1/tnh10s 1 71.pdf

SENASA. (2014). Biología y comportamiento de la mosca dela fruta. Obtenido dehttps://www.senasa.gob.pe/senasa/wpcontent/uploads/2014/12/Biologia-de-laMosca.pdf

Tigrero, J. O. (2009). Lista anotada de hospederos de moscas de la fruta presentes en el Ecuador. Serie Zoológica5(9), 107-116. Obtenidodehttp://www.programamoscamed. $\mathrm{mx} /$ EIS/biblioteca/libros/articulos/Tigrero.\% 20 2009.pdf

Torres, S. J., \& Ríos, N. (2015). Identificación de las principales especies y hospedero de mosca dela fruta en Marcabal, Provincia de Sánchez Carrión, La Libertad. tes is. Obtenidodehttp://dspace.unitru.edu.pe/bitstr eam/handle/UNITRU/4157/TORRES\%20C ASTILLO $\% 20$ Saul.pdf? sequence $=1$ uence= 1

Vilatuña, J., Valenzuela , P., Bolaños, J., Hidalgo , R., \& Mariño , A. (2016). Hospederos de la mosca de la fruta Anastrepha spp. Y Ceratitis capitata (Diptera: Tephiridae) en Ecuador. Científica Ecuatoriana,3. Obtenido de https://www.google.com.pe/url?sa=t\&rct $=$ j $\& \mathrm{q}=\&$ es $\mathrm{rc}=\mathrm{s} \& \mathrm{~s}$ ource $=\mathrm{web} \& \mathrm{~cd}=1 \& \mathrm{c}$ $\mathrm{ad}=$ rja\&uact $=8 \&$ ved $=0$ ahUKEwi $5 \mathrm{au} 4 \mathrm{oKD}$ VA h UB 5 S Y K H c 8 H B jw Q F g g n M A A\&url=http $\% 3 \mathrm{~A} \% 2 \mathrm{~F} \% 2 \mathrm{Fwww}$.agrocalidad. gob.ec $\% 2$ Frevistaecuadorescalidad $\% 2$ Finde x.php $\% 2$ Frevista $\% 2$ Farticle $\% 2$ Fdownload $\%$ 2F31\%2F68\&usg=AFQjC 